\title{
Valor en riesgo en el sector petrolero: un análisis de la eficiencia en la medición del riesgo de la distribución $\alpha$-estable versus las distribuciones t-Student generalizada asimétrica y normal
}

\author{
Value at risk in the oil sector: An analysis of the efficiency in the measurement of \\ the risk of the $\alpha$-stable distribution versus the generalized asymmetric Student- $t$ \\ and normal distributions
}

\author{
Ramona Serrano Bautista ${ }^{1 *}$, José Antonio Núñez Mora ${ }^{2}$ \\ ${ }^{1}$ Universidad Panamericana, Escuela de Ciencias Económicas y Empresariales, México \\ ${ }^{2}$ Instituto Tecnológico y de Estudios Superiores de Monterrey, EGADE Business School, México
}

Recibido el 26 de abril de 2018; aceptado el 14 de diciembre de 2018

Disponible en Internet el: 9 de enero de 2019

\section{Resumen}

En el sector petrolero, el VaR se ha implementado con el objetivo de cuantificar lo mejor posible los movimientos extremos de los precios del petróleo, debido a que estos repercuten la actividad económica y afectan significativamente los movimientos en el mercado accionario (Sadorsky, 1999). Con este propósito, en esta investigación cuantificamos el VaR considerando tres tipos de petróleo (Brent, WTI y MME) y analizamos el desempeño de la estimación del VaR a un día mediante el estadístico de Kupiec considerando modelos GARCH con tres distribuciones alternativas en el proceso de innovación: estable, t-Student generalizada asimétrica y normal en un período de alta volatilidad. Los resultados de la evaluación de desempeño del modelo basado en el estadístico de Kupiec señalan que el modelo VaR-estable es un modelo más robusto y preciso para ambos niveles de confianza que los basados en las distribuciónes t-Student generalizada asimétrica y normal. Este resultado es crucial en el sector financiero, debido a que impacta directamente en la previsión de reservas necesarias para afrontar potenciales pérdidas.

\footnotetext{
*Autor para correspondencia.

Correo electrónico ramserrano77@gmail.com (R. Serrano Bautista).

La revisión por pares es responsabilidad de la Universidad Nacional Autónoma de México. 
Código JEL: G17, C22, C13

Palabras clave: Distribución estable; Distribución t-Student generalizada asimétrica; Valor en riesgo (VaR); GARCH

\begin{abstract}
In the oil sector, value at risk (VaR) can be used to quantify as best as possible the maximum oil price changes, because these have an impact on economic activity and finds evidence of its importance in explaining movements in the stock returns (Sadorsky, 1999). With this purpose, in this paper we quantify the VaR of three types of oil (Brent, WTI and MME) and analyze the performance of the one-day VaR estimation by Kupiec test considering GARCH models with three alternative distributions in the innovation process: stable, Student-t generalized and normal in a period of high volatility. The results of the performance evaluation of the model based on the Kupiec statistic indicate that the VaR-stable model is a more robust and accurate model for both confidence levels than those based on the generalized asymmetric and normalized Student t-distributions. This result is crucial in the financial sector, because it directly impacts the provision of reserves necessary to face potential losses.
\end{abstract}

JEL codes: G17, C22, C13

Keywords: Stable distributions; Generalized skew t distribution; Value at risk (VaR); GARCH

\title{
Introducción
}

A partir de la liberación del sector energético en 1970, los precios del petróleo y su elevada volatilidad han generado una gran preocupación para los consumidores, los productores y los gobiernos, así como un creciente interés académico por estudiar esta importante variable económica en la administración del riesgo. Diversos factores afectan el precio del petróleo, los principales son: las políticas de la Organización de Países Exportadores de Petróleo (OPEP) ${ }^{1}$, conflictos militares, tensiones geopolíticas, desastres naturales, desequilibrios entre la oferta y la demanda en los mercados internacionales, entre otros.

El petróleo es un insumo fundamental en la economía mundial, debido a que es una fuente primaria de energía en el sector industrial, eléctrico y de transporte. Hamilton (1983), argumenta que los movimientos extremos de los precios del petróleo son parcialmente responsables de las recesiones ocurridas entre 1948 y 1972 en Estados Unidos. Por otro lado, Sadorsky (1999), afirma que los movimientos extremos en los precios del petróleo repercuten la actividad económica y afectan significativamente los movimientos en el mercado accionario.

${ }^{1}$ La OPEP controla aproximadamente el 43\% de la producción mundial de petróleo y el 81\% de las reservas de petróleo. 
Por lo cual, bajo este ambiente de incertidumbre y volatilidad es esencial contar con modelos que describan lo mejor posible las fluctuaciones de los precios del petróleo con el objetivo de implementar una herramienta eficiente en la administración del riesgo derivado de los movimientos extremos en los precios del petróleo. El Valor en Riesgo (VaR) se ha convertido en el estándar para medir y evaluar el riesgo en los mercados financieros, debido a la simplicidad de su interpretación. El VaR se define como la máxima pérdida probable de un portafolio o instrumento financiero en un horizonte temporal determinado, para un nivel de confianza dado, bajo circunstancias normales de los mercados y como consecuencia de movimientos adversos de los precios.

El objetivo de la presente investigación es estimar un modelo GARCH-estable para pronosticar la volatilidad de los precios del petróleo e implementarlo en la estimación del VaR. Además, mediante el estadístico de Kupiec se analiza el desempeño de la estimación del VaR a un día considerando modelos GARCH con distribuciones alternativas a la estable en el proceso de innovación como la normal y t-Student generalizada asimétrica (GST).

Es importante mencionar que en la estimación del VaR, la elección de la distribución apropiada del proceso de innovación es crucial ya que impacta directamente en la calidad de la estimación de los cuartiles requeridos para estimar el riesgo, además, el supuesto sobre la distribución en el modelo GARCH es también clave en el pronóstico del VaR, ya que en base a éste se construyen las funciones de verosimilitud requeridas para estimar los parámetros y se determina la distribución futura del riesgo condicionada a la volatilidad pronosticada.

El resto del documento se organiza de la siguiente forma: En la Sección 2 se realiza la revisión de la literatura sobre la medición del riesgo en el sector petrolero, en la Sección 3 se describe el enfoque GARCH basado en la distribución estable y GST, la metodología de las estimaciones del VaR y las pruebas de desempeño del mismo. Los datos y su análisis preliminar se presentan en la Sección 4. Los resultados empíricos son descritos en la Sección 5 y el documento finaliza con las conclusiones presentes en la Sección 6.

\section{Revisión de la literatura}

Actualmente la literatura sobre la medición del riesgo en el sector petrolero es escasa, a pesar de la gran necesidad de gestionar el riesgo derivado de los movimientos del precio del petróleo.

Con el objetivo de ofrecer una visión comparativa, en esta sesión se resumen los principales estudios en la literatura que demuestran que los modelos tipo GARCH son herramientas ampliamente utilizadas en la literatura para analizar la volatilidad y el VaR en el sector petrolero.

Morana (2001) analiza la aplicabilidad del GARCH semiparamétrico propuesto por Barone-Adesi et al. (1999) para pronosticar la distribución de los precios del petróleo Brent en horizontes a corto plazo. Los resultados señalan que los pronósticos fuera de la muestra 
sugieren que el GARCH semiparamétrico puede ser empleado para estimar el VaR bajo diferentes horizontes de tiempo.

En la misma línea Costello et al. (2008) analiza el desempeño del modelo de simulación histórica con predicciones ARMA (HSAF) y el modelo GARCH semiparamétrico propuesto por Barone-Adesi et al. (1999) en la estimación del riesgo del petróleo Brent. Los hallazgos señalan que las estimaciones del VaR mediante el modelo semiparamétrico son superiores a las obtenidas mediante el modelo HSAF.

En contraste, Cabedo y Moya (2003) proponen el uso del VaR en la cuantificación del riesgo en el mercado petrolero del Brent. Estiman el VaR mediante simulación histórica (HS), simulación histórica con predicciones ARMA (HSAF) y método paramétrico basado en el GARCH bajo la hipótesis normal, los resultados señalan que la metodología HSAF proporciona una mejor estimación del VaR en términos porcentuales.

De manera similar, Sadhegi y Shavvalpour (2006) comparan el desempeño del modelo HSAF y el método paramétrico basado en el modelo GARCH bajo la hipótesis normal en la estimación del VaR, a diferencia de las investigaciones previamente mencionadas, emplean precios semanales de la mezcla OPEC. Concluyen que el modelo HSAF es más eficiente en la estimación del VaR.

Por otro lado, Giot y Laurent (2003) evalúan el desempeño del método RiskMetrics, el modelo APARCH${ }^{2}$ y ARCH ambos bajo la hipótesis t-Student asimétrica en la estimación del riesgo del petróleo Brent, WTI, del aluminio, cobre, niquel y de los contratos futuros sobre el cacao. Los resultados señalan que el modelo APARCH se desempeña mejor en todos los casos, sin embargo, el modelo ARCH provee excelentes resultados en la estimación del VaR y su ventaja es su fácil implementación.

Por su parte, Sadorsky (2006) analiza el ajuste de diferentes modelos estadísticos univariados y multivariados en la estimación de la volatilidad de los precios del petróleo WTI, heating oil \#2, gasolina sin plomo y gas natural y compara las estimaciones del VaR paramétricas y no paramétricas. Los resultados señalan que en el caso de los futuros del petróleo que el modelo GARCH es el que mejor ajuste presenta en la estimación de la volatilidad, sin embargo, considerando el número de excedencias del VaR los modelos no paramétricos superan a los modelos paramétricos.

Hung et al. (2008) comparan la precisión y eficiencia de las estimaciones del VaR mediante el modelo GARCH bajo la hipótesis normal (GARCH-N), t-student (GARCH-t) y la distribución de colas pesadas (GARCH-HT) para el petróleo Brent, WTI, heating oil \#2, propano y gasolina NYHCGR. Los hallazgos encontrados son que las estimaciones del VaR basadas en el GARCH-HT poseen una mejor precisión tanto para niveles de confianza bajos y altos.

Fan et al. (2008) realizan estimaciones del VaR del petróleo Brent y WTI mediante los

${ }^{2}$ Modelo de heterocedasticidad condicional autorregresivo de potencia asimétrica. 
modelos GARCH bajo la hipótesis de la distribución Generalizada del Error (GARCH-GED), la hipótesis normal (GARCH-N) y a través del modelo HSAF y comparan su respectiva eficiencia. Concluyen que la estimación del VaR a través del GARCH-GED es más eficiente.

Marimoutou et al. (2009) estiman el VaR aplicando la Teoría de Valores Extremos (EVT) condicional e incondicionalmente y comparan el desempeño de estos modelos con los modelos de simulación histórica, HSAF y algunos modelos paramétricos basados en la estimación del GARCH para el caso del petróleo Brent y WTI. Los resultados señalan que los modelos EVT condicional y HSAF tienen un desempeño superior que el resto de los modelos comparados.

Aloui y Mabrouk (2010) comparan la habilidad de algunos modelos GARCH de memoria larga para predecir la volatilidad de los precios del petróleo WTI, Brent y de las gasolinas NYHCGR y RCGR. Además, evalúan el desempeño del VaR paramétrico considerando tres distribuciones: normal, t-student y $\mathrm{t}$-student asimétrica. Concluyen que el modelo FIGARCH bajo la hipótesis t-student asimétrica muestra un mejor desempeño en la predicción del VaR que el resto de los modelos comparados.

Cheng y Hung (2011) estiman el VaR paramétrico condicional considerando la distribución t-student asimétrica generalizada (GST), la distribución Generalizada del Error (GED) y la distribución normal, la muestra incluye los precios diarios del petróleo WTI, gasolina NYHCGR, heating oil \#2, oro, plata y cobre. Las pruebas de cobertura condicional e incondicional señalan que la estimación del VaR bajo la hipótesis t-student asimétrica generalizada muestra un mejor desempeño.

Youssef et al. (2015) comparan la habilidad de algunos modelos GARCH de memoria larga para predecir la volatilidad de los precios de algunos energéticos entre ellos el petróleo WTI y Brent y posteriormente aplicarlos en la estimación del VaR aplicando la Teoría de Valores Extremos. Concluyen que las estimaciones del VaR bajo el método FIAPARCH-EVT son más precisas.

De Jesús Gutierrez et al. (2016) aplican la Teoría de Valores Extremos en la estimación del VaR y CVaR para el caso de la mezcla de petróleo mexicana (MME) considerando algunos modelos GARCH y comparan estas estimaciones con las obtenidas mediante HS Y HSAF. El estadístico de Kupiec evidencia que los modelos TVE condicional y HSAF presentan el mejor desempeño en la estimación del VaR condicional de las posiciones corta y larga en cualquier nivel de confianza, aunque su desempeño se reduce significativamente en la predicción del CVaR.

Continuando con el caso mexicano, Ruiz-Porras y Anguiano Pita (2016) analizan el caso multivariado y describen las volatilidades e interrelaciones de los rendimientos del petróleo Brent, WTI y MME mediante 12 modelos GARCH. Entre sus hallazgos señalan que el modelo $\operatorname{AR}(1)-\operatorname{TGARCH}(1,1)$ bajo la hipotesis t-Student multivariada genera un ajuste satisfactorio para los rendimientos dentro de esta familia de modelos. 
La presente investigación difiere de la literatura previa en al menos dos puntos: en primer lugar, hasta donde es de nuestro conocimiento, los modelos GARCH-estable no se han implementado para pronosticar la volatilidad de los precios del petróleo. Hasta hoy la distribución estable se ha aplicado en el análisis de riesgo en el mercado accionario, pero las características empíricas de las series de rendimientos del petróleo como lo son colas pesadas, asimetría y clúster de volatilidad, sugieren que el modelo GARCH-estable es un modelo adecuado para capturar estas características empíricas de manera eficiente; en segundo lugar, analizamos el desempeño de la estimación del VaR a un día mediante el estadístico de Kupiec considerando modelos GARCH con distribuciones alternativas a la estable como la distribución t-Student generalizada asimétrica ${ }^{3}$ y la distribución normal.

\section{Metodología}

En esta sección, se describe la metodología aplicada para analizar el riesgo de los rendimientos de los petróleos Brent, WTI Y MME.

\section{Valor en Riesgo (VaR)}

Jorion (2001) define el Valor en Riesgo como la máxima pérdida esperada en un horizonte temporal determinado, para un nivel de confianza dado.

Definición. Dada X una variable aleatoria continua definida sobre el espacio muestral $\boldsymbol{\Omega}$ que representa el cambio en el valor del activo (rendimiento). Supongamos que $X: \Omega \rightarrow \mathbb{R}$, está definida sobre un espacio de probabilidad fijo $(\Omega, \mathrm{A}, P)$. Entonces, el Valor en Riesgo de $X$ al nivel 1-q se define como la mínima de las cotas superiores para un intervalo de confianza del (1-q) \% tal que

$$
P\left\{X \leq-\operatorname{VaR}_{1-q}^{X}\right\}=q
$$

Esta definición señala que es posible obtener el VaR dada la función de distribución acumulada de los rendimientos del activo:

$$
-\operatorname{VaR}_{1-q}^{X}=F_{X}^{-1}(q)
$$

donde $F_{X}^{-1}(\cdot)$ es la inversa de la función de distribución acumulada de los rendimientos del activo en un período. Es decir, el VaR es el cuantil $q$ de $F x$. Por lo cual, la esencia de los cálculos del VaR es la estimación de los cuantiles inferiores de la función de distribución acumulada de los rendimientos del activo, que en la práctica es desconocida.

\footnotetext{
${ }^{3}$ Cheng y Hung (2011) señalan basados en pruebas de cobertura condicional e incondicional que la estimación del VaR bajo la hipótesis t-Student asimétrica generalizada muestra un mejor desempeño en el mercado de los energéticos.
} 
Los métodos de estimación del VaR sugieren diferentes formas de construir esta función. Los más comunes son, el método paramétrico basado en el supuesto de que los cambios en el valor del portafolio son caracterizados por una distribución paramétrica; la simulación histórica en cuya implementación no es necesario asumir una distribución específica de los rendimientos, las predicciones de su comportamiento se infieren empleando el comportamiento histórico de los datos; y la simulación Montecarlo en la cual se obtienen aproximaciones del comportamiento del rendimiento esperado de un portafolio o instrumento financiero, mediante simulaciones que generan trayectorias aleatorias de los rendimientos del portafolio o instrumento financiero, considerándose ciertos supuestos iniciales sobre las volatilidades y correlaciones de los factores de riesgo.

En el presente trabajo la estimación del VaR se realiza mediante simulación Montecarlo, bajo la hipótesis estable, t-Student generalizada asimétrica y normal.

\section{Distribución $\alpha$-estable y $t$-Student Generalizada Asimétrica}

En la gestión del riesgo es esencial encontrar una distribución que describa los datos financieros de una manera adecuada. Comúnmente los rendimientos financieros no son descritos adecuadamente por la distribución normal, ya que los resultados empíricos muestran que los datos financieros son por lo general asimétricos y presentan colas pesadas (Fama, 1965; Bollerslev, 1986; Bali y Theodossiou, 2007; Champagnat et al., 2013). Sin embargo, esta característica no es exclusiva de los activos financieros, las series de rendimientos de los energéticos también comparten estas características (Giot y Laurent, 2003; Hang et al., 2007; Fan et al., 2008).

Actualmente, la mayoría de los estudios que estiman la volatilidad de los rendimientos del petróleo lo hacen bajo la hipótesis gaussiana (Sadeghu y Shavvalpour, 2006). La literatura existente relativa a la estimación de la volatilidad en el sector energético aplicando distribuciones alternativas a la normal es muy escasa (Giot y Laurent, 2003; Hang et al., 2007; Fan et al., 2008; Hung et al., 2008; Marimoutou et al., 2009; Aloui y Mabrouk, 2010; Cheng y Hung, 2011; Youssef et al., 2015).

En este trabajo presentamos dos familias de distribuciones: la distribución estable y la distribución t-Student generalizada asimétrica (GST), los cuales nos permiten describir las características de asimetría y curtosis de los rendimientos en el sector petrolero.

\section{Distribución t-Student Generalizada Asimétrica}

Existen diversas parametrizaciones de la distribución t-student generalizada asimétrica propuestas en investigaciones previas, en este estudio debido a su simplicidad, seguimos la parametrización propuesta por Hansen (1994). Este sugiere un enfoque paramétrico alternativo para modelar la función de densidad condicional del error normalizado. Este consiste en 
seleccionar una distribución que dependa de un vector de parámetros de pocas dimensiones, y permitir que este vector varíe en función de las variables condicionales.

Definición. La distribución t-Student generalizada asimétrica (GST) es la generalización de la distribución t-student, la cual considera asimetría. La función de densidad de probabilidad de la distribución estándar GST se define como:

$$
f(z ; \eta, \lambda)= \begin{cases}b c\left(1+\frac{1}{\eta-2}\left(\frac{b z+a}{1-\lambda}\right)^{2}\right)^{-\frac{\eta+1}{2}} & z<-\frac{a}{b} \\ b c\left(1+\frac{1}{\eta-2}\left(\frac{b z+a}{1+\lambda}\right)^{2}\right)^{-\frac{\eta+1}{2}} & z \geq-\frac{a}{b}\end{cases}
$$

donde $2<\eta<\infty,-1<\lambda<1, a=4 \lambda c \frac{\eta-2}{\eta-1}, b=\sqrt{1+3 \lambda^{2}-a^{2}}$ у $c=\frac{\Gamma\left(\frac{\eta+1}{2}\right)}{\sqrt{\pi(\eta-2) \Gamma\left(\frac{\eta}{2}\right)}}$.

En Hansen (1994) se demuestra que esta es una función de densidad apropiada con media 0 y varianza 1 . El parámetro $\eta$ controla el grosor de la cola y $\lambda$ controla la asimetría. Cuando $\eta \rightarrow \infty$, la distribución se reduce a la distribución normal asimétrica. Cuando $\lambda=0$, se reduce a la distribución t-student.

Si una variable aleatoria Z sigue una distribución estándar GST con parámetros $\eta$ y $\lambda$, se denota como Z $\square$ GST $(\eta, \lambda)$. Si la variable aleatoria $Z$ sigue una distribución no estándar GST con media $\mu$ y varianza $\sigma 2$ se denota como Z $\square \operatorname{GST}(\mu, \sigma, \eta, \lambda)$.

\section{Distribución $\alpha$-estable}

La familia de distribuciones $\alpha$-estable o simplemente estables es una clase de distribuciones de probabilidad que permiten asimetría y colas pesadas. En 1920, el matemático francés Paul Lévy caracterizó esta clase de distribuciones en su estudio de sumas de términos independientes idénticamente distribuidos. Las distribuciones estables no poseen expresiones analíticas explícitas para la función de densidad de probabilidad (PDF) ni para la función de distribución acumulada (CDF), sin embargo estas son descritas por su función característica (CF).

Definición. Una variable aleatoria $\alpha$-estable X, es comúnmente descrita por su función característica $(\mathrm{CF})$, la cual se define como:

$$
\begin{aligned}
\Phi_{X}(t ; \alpha, \beta, \gamma, \delta)= & E[\exp (i X t) \\
& = \begin{cases}\exp \left(-\gamma^{\alpha}|t|^{\alpha}\left[1-i \beta \operatorname{sgn}(t) \tan \left(\frac{\pi \alpha}{2}\right)\right]+i \delta t\right), & \alpha \neq 1 \\
\exp \left(-\gamma|t|\left[1+i \beta \frac{2}{\pi} \operatorname{sgn}(t) \ln |t|\right]+i \delta t\right), & \alpha=1\end{cases}
\end{aligned}
$$


donde, $\operatorname{sgn}(t)=\{1$ si $t>0,0$ si $t=0,-1$ si $t<0\}, 0<\alpha \leq 2$ es el índice de estabilidad o exponente característico que nos refleja el tamaño de las colas de la distribución, $-1 \leq \beta \leq 1$ es el parámetro de asimetría que nos índica la simetría de la distribución, $\gamma \geq 0$ es un parámetro de escala también denominado dispersión, $\delta \in \mathrm{R}$ y es el parámetro de posición.

Si una variable aleatoria $\mathrm{Z}$ sigue una distribución estable se denota como $\mathrm{Z} \square \mathrm{S}(\alpha, \beta, \gamma, \delta)$ entonces una variable aleatoria $\alpha$-estable estándar se denota como $\mathrm{Z} \square \mathrm{S}(\alpha, \beta, 1,0)$.

\section{Modelos GARCH}

La volatilidad es la variable principal sobre la que se desarrollan los modelos económicos y financieros de fijación de precios y coberturas, por lo cual realizar estimaciones con las especificaciones correctas de la distribución condicional es crucial para mejorar la eficiencia de los mismos. En este trabajo, implementamos el $\operatorname{GARCH}(1,1)$ bajo la hipótesis estable, GST y normal para describir la volatilidad de los rendimientos petroleros. Sadorsky (2006) afirma que el modelo GARCH $(1,1)$ posee un buen desempeño en la estimación de la volatilidad en el sector petrolero.

\section{Modelo GARCH(1,1)-estable}

Para describir las características empíricas de los rendimientos del petróleo como lo son colas pesadas, asimetría y clúster de volatilidad, empleamos la distribución estable para describir el proceso de innovación en el modelo $\operatorname{GARCH}(1,1)$. Debido a que en el caso de la distribución estable no todos los momentos están definidos, empleamos el modelo TS-GARCH propuesto por Taylor (1986) y Schwert (1989), en el cual los rendimientos son modelados como se describe a continuación:

$$
\begin{gathered}
R_{t}=\delta_{t}+\varepsilon_{t} \\
\varepsilon_{t}=\gamma_{t} z_{t} \\
\left|\gamma_{t}\right|=a_{0}+a_{1}\left|\varepsilon_{t-1}\right|+b_{1}\left|\gamma_{t-1}\right|
\end{gathered}
$$

donde $R_{t}$ es la serie de rendimientos de la acción en el tiempo $t, \delta_{t} y \gamma_{t}$ son los parámetros de posición y de dispersión condicional respectivamente, y $z_{t}$ son variables aleatorias estandarizadas estable idéntica e independientemente distribuidas, $z_{t} \sim S(\alpha, \beta, 1,0) 1<\alpha<2$.

Los parámetros son estimados por máxima verosimilitud, empleando el programa STABLE descrito en Nolan (1997).

\section{Modelo $\operatorname{GARCH}(1,1)-G S T$}

Similarmente, empleamos la distribución GST en el modelo GARCH para describir los hechos estilizados característica de la serie de rendimientos petroleros. El modelo es descrito a continuación: 


$$
\begin{gathered}
R_{t}=\mu_{t}+\varepsilon_{t} \\
\varepsilon_{t}=\sigma_{t} z_{t} \\
\sigma_{t}^{2}=a_{0}+a_{1}\left(\varepsilon_{t-1}-c\right)^{2}+b_{1} \sigma_{t-1}^{2}
\end{gathered}
$$

Para estimar los parámetros del modelo GARCH, utilizamos el método de máxima verosimilitud (MLE), donde la función de densidad de probabilidad de zt se aproximó utilizando el enfoque propuesto por Hansen(1994).

\section{Modelo GARCH(1,1)-normal}

En este modelo, los rendimientos de las acciones son modelados como:

$$
\begin{gathered}
R_{t}=\mu_{t}+\varepsilon_{t} \\
\varepsilon_{t}=\sigma_{t} z_{t} \\
\sigma_{t}^{2}=a_{0}+a_{1} \varepsilon_{t-1}^{2}+b_{1} \sigma_{t-1}^{2}
\end{gathered}
$$

Evaluación del desempeño del VaR

En esta investigación la evaluación del desempeño del VaR se realiza en términos de su probabilidad de cobertura empírica, mediante el estadístico de Kupiec (1995). Este estadístico es una prueba incondicional que cuenta el número de violaciones del VaR durante todo el período, estimando si la proporción esperada de violaciones es igual al nivel de significancia $\alpha$.

El estadístico de prueba de Kupiec para muestras grandes se distribuye como una Ji-cuadrada con un grado de libertad y está dado por:

$$
L R_{U C}=-2 \ln \left[\frac{\alpha^{n}(1-\alpha)^{T-n}}{p^{n}(1-p)^{T-n}}\right]
$$

donde $T$ representa el tamaño de la muestra, $\mathrm{n}$ el número de violaciones y $p=n / T$ es el porcentaje de violaciones. La hipótesis nula $H_{0}: n / T=\alpha$, se rechaza con un nivel de significancia del $1 \%$ si el valor del estadístico de Kupiec excede o es igual al valor crítico de una distribución Ji-cuadrada con un grado de libertad, es decir $\mathrm{LR}_{\mathrm{UC}} \geq 6.635$.

\section{Datos y análisis preliminar}

En esta sección se describe la base de datos, se muestran las estadísticas descriptivas, pruebas de raíz unitaria, pruebas de efectos ARCH y pruebas de bondad de ajuste de las respectivas distribuciones. Las figuras, tablas y rutinas algebraicas requeridas en esta investigación fueron programadas en MATLAB R2017a. 


\section{Descripción de los datos y análisis estadístico}

El presente trabajo utiliza los precios diarios de cierre del petróleo West Texas Intermediate (WTI), del Mar del Norte (Brent) y de la mezcla de petróleo mexicana de exportación (MME), la muestra cubre el período del 2 de enero del 2013 al 29 de diciembre del 2017, obteniendo un total de 1275 observaciones y la moneda de referencia es el dólar estadounidense. Las series de precios fueron obtenidas de Bloomberg en el caso de la mezcla de petróleo mexicana y en caso del WTI y Brent se obtuvieron del sitio web Energy Information Administration (EIA).

Se seleccionaron estas tres mezclas de petróleo debido a que en el caso del Brent su precio es el referente en los mercados europeos, el WTI es el más aceptado como referencia mundial del precio del barril de petróleo y el MME es el referente en el mercado mexicano.

El período de la muestra se seleccionó por dos razones: primero analizar el desempeño del VaR bajo las distintas funciones de distribución consideradas en este trabajo en períodos de alta volatilidad y segundo hasta donde es de nuestro conocimiento no existe referencia alguna de la medición del VaR en este período específico, para este tipo de crudos bajo la hipótesis de estabilidad. Además, durante este período se registró un severo desplome de los precios del crudo, los cuales experimentaron la tercera mayor depreciación semestral de los últimos 24 años. El World Bank (Global Economic Prospects January 2015) identificó cuatro razones para la caída de los precios del petróleo 2014-2015 señalando a los primeros tres factores como dominantes: 1) el exceso de oferta en un momento de debilitamiento de la demanda, 2) un cambio en los objetivos de la OPEP, 3) la disminución de la preocupación en torno a las interrupciones de suministro por causas geopolíticas, y 4) la apreciación del dólar estadounidense.

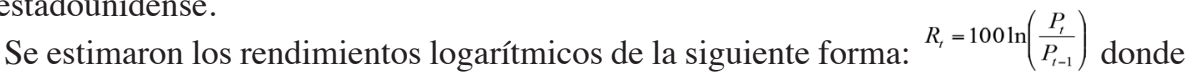
$P_{t}$ es el precio en el día $t$. En la figura 1 se muestra la gráfica del comportamiento de los precios diarios de cierre de los petróleos y sus respectivos rendimientos logarítmicos. En esta figura se observa que las series de rendimientos logarítmicos muestran heterocedasticidad y clusters de volatilidad. 

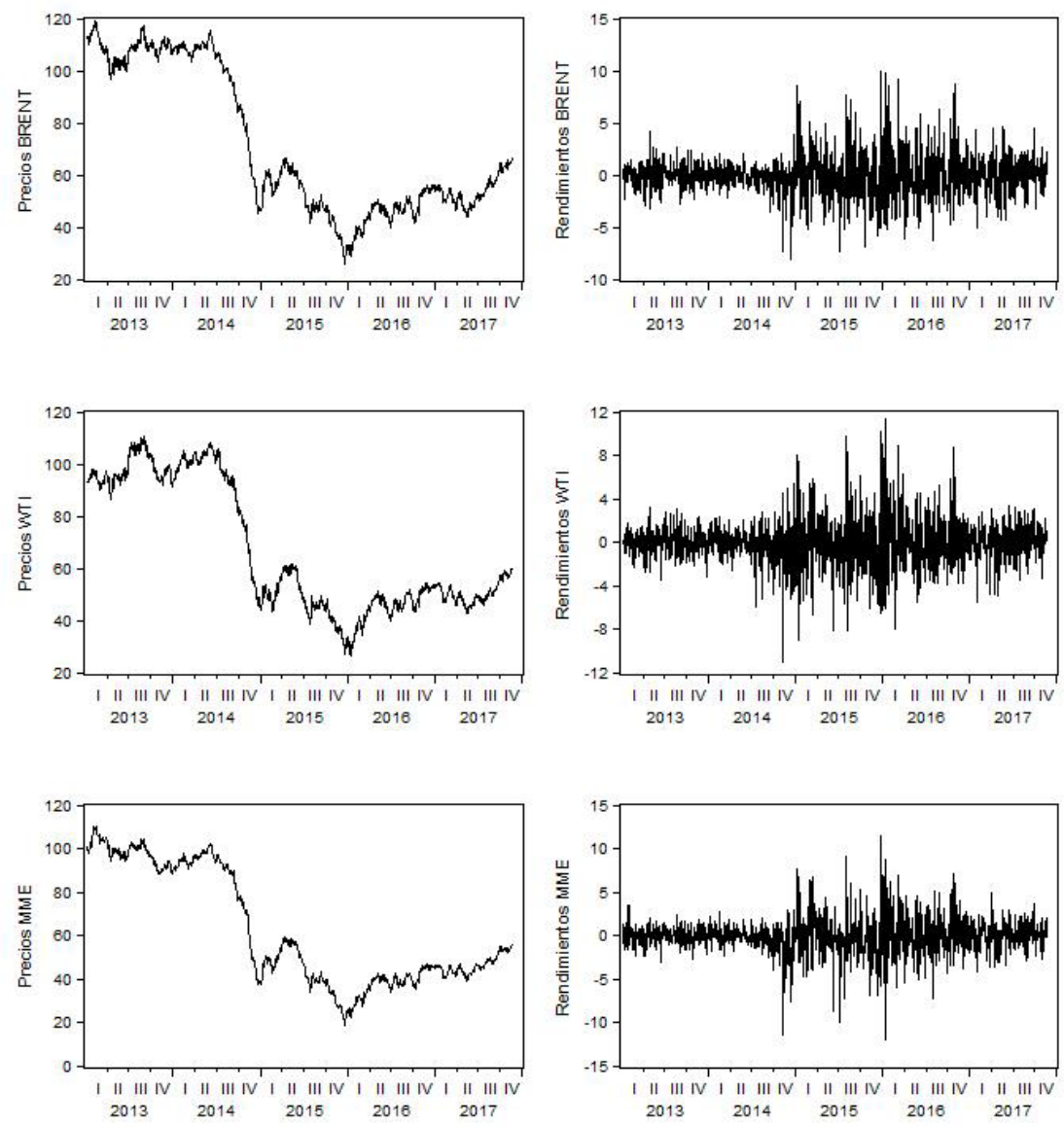

Figura 1. Precios diarios y rendimientos logarítmicos.

Fuente: Elaboración propia.

En la Tabla 1 se muestra la estadística descriptiva de los rendimientos, se contrasta la normalidad de los mismos y se presentan las pruebas de raíz unitaria, de estacionariedad y de efectos ARCH. Se observa que las medias de los tres rendimientos son pequeñas, negativas y muestran valores similares, en contraste la desviación estándar correspondiente es alta en comparación a la media, y además la curtosis indica un comportamiento leptocúrtico de las series. Los estadísticos Jarque-Bera (1980), Shapiro-Wilks (1965) y Anderson-Darling rechazan la hipótesis de normalidad en las series y las pruebas de raíz unitaria ADF (Dickey-Fuller, 1979), y PP (Phillips-Perron, 1988), rechazan la hipótesis de raíz unitaria para las series de tiempo estudiadas, es decir las series son estacionarias. Además, los resultados de la prueba 
KPSS (Kwiatkowski, Phillips, Schmidt and Shin, 1992) revelan que no podemos rechazar la hipótesis nula de estacionariedad en tendencia determinística en un nivel significativo del $1 \%$, es decir todas las series presentan estacionariedad en tendencia. Finalmente, la prueba ARCH-LM rechaza la hipótesis de la no existencia de efectos ARCH (Engle, 1982), es decir las series de rendimientos estudiadas manifiestan efectos ARCH.

Tabla 1

Estadística descriptiva, pruebas de normalidad, raíz unitaria y estacionariedad

\begin{tabular}{lccc}
\hline Series & BRENT & WTI & MME \\
\hline Panel A: Estadística descriptiva & & \\
Media & -0.0413 & -0.0339 & -0.0449 \\
Dev. Std. & 2.0057 & 2.2014 & 1.9964 \\
Asimetría & 0.4222 & 0.2113 & 0.0150 \\
Curtosis & 6.1537 & 6.2773 & 8.2532
\end{tabular}

Panel B: Pruebas de normalidad

$\begin{array}{lccc}\text { Jarque-Bera } & 565.80 * & 579.64 *(0.0000) & 1464.96 *(0.0000 \\ & (0.0000) & & 8.7466^{*} \\ \text { Shapiro-Wilks } & 6.2800 * & 6.7707 * & (0.0000) \\ & (1.6934 \mathrm{e}-10) & (6.4069 \mathrm{e}-12) & 9.0263 * \\ \text { Anderson-Darling } & 4.7404 * & 2.8404 * & (5.0000 \mathrm{e}-04) \\ & & & \end{array}$

Panel C: Pruebas de raíz unitaria y estacionariedad

$\begin{array}{lccc}\text { ADF } & -34.1951^{*} & -37.7924 *(0.0000) & -30.6914 * \\ & (0.0000) & & (0.0000) \\ \text { PP } & -34.2847 * & -37.7934 *(0.0000) & -30.9370 * \\ & (0.0000) & & (0.0000) \\ \text { KPSS } & 0.2614 & 0.2113 & 0.2422\end{array}$

Panel D: Prueba de efectos ARCH

$\begin{array}{llll} & 12.4524 * & 11.1306 *(0.0000) & 6.1016 *(0.0000) \\ \text { ARCH-LM } & (0.0000) & & \end{array}$

Las pruebas de raíz unitaria ADF y PP incluyen tendencial lineal e intercepto. La prueba de efectos ARCH se realiza considerando 5 rezagos. Los p-values se muestran entre paréntesis y $*$ indica significancia a un nivel de $1 \%$. Fuente: Elaboración propia. 


\section{Pruebas de bondad de ajuste}

Para comparar la bondad de ajuste de las distribuciones alternativas consideradas en este documento se considera la prueba de Kolmogorov-Smirnov (KS)), cuya hipótesis nula es H0: Los datos analizados siguen la distribución señalada. La hipótesis nula no se rechaza si el p-value excede o es igual al nivel de significancia elegido.

La Tabla 2 muestra los valores del estadístico de contraste de la prueba KS y su respectivo p-value, los cuales indican un no rechazo de la hipótesis nula con un nivel de significancia $\alpha=.01$, excepto para la serie MME en el caso de la distribución GST.

Tabla 2

Bondad de ajuste

\begin{tabular}{|c|c|c|}
\hline Distribuciones & Estable & GST \\
\hline & $\mathrm{KS}$ & $\mathrm{KS}$ \\
\hline BRENT & $0.0331(0.1206)$ & $0.0400 \quad(0.0333)$ \\
\hline WTI & $0.0169(0.8594)$ & $0.0318 \quad(0.1493)$ \\
\hline MME & $0.0395(0.0365)$ & $0.0529 \quad(0.0015)$ \\
\hline
\end{tabular}

Los p-values se muestran entre paréntesis.

Fuente: Elaboración propia.

\section{Estimación de los parámetros de las distribuciones de probabilidad}

Los parámetros de las distribuciones alternativas consideradas, se estimaron por el método de máxima verosimilitud (MLE). La Tabla 3 muestra los valores obtenidos.

Tabla 3

Estimación de los parámetros de las distintas distribuciones alternativas.

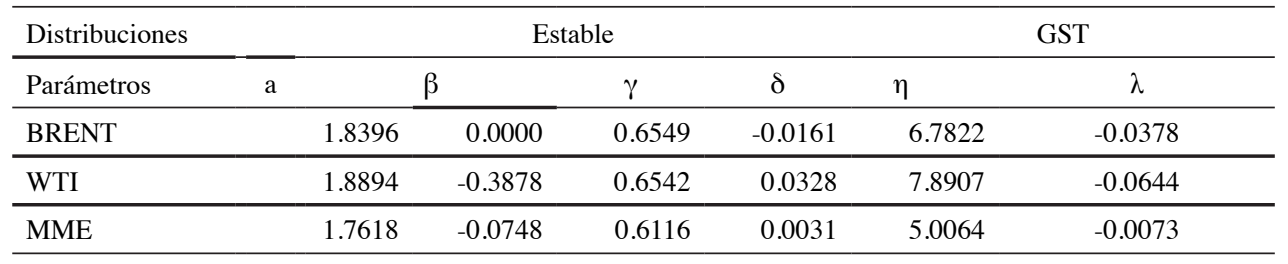

Los intervalos de confianza al 95\% se muestran entre paréntesis.

Fuente: Elaboración propia.

Resultados empíricos

Estimación de los modelos GARCH 
Para describir las características empíricas de los rendimientos del petróleo como lo son colas pesadas, asimetría y clúster de volatilidad implementamos el modelo $\operatorname{GARCH}(1,1)$ bajo la hipótesis estable, GST y normal. Los parámetros de los modelos GARCH son estimados por MLE y son significativos al 1\%, estas estimaciones se muestran en la Tabla 4.

Tabla 4

Estimación de los parámetros de los modelos GARCH

\begin{tabular}{lllll}
\hline Parametros & $a_{0}$ & $a_{1}$ & $c$ & $b_{1}$ \\
\hline Estable & & & \\
\hline BRENT & 0.0258 & 0.0646 & - & 0.9352 \\
\hline WTI & 0.0329 & 0.0746 & - & 0.9252 \\
\hline MME & 0.0207 & 0.0488 & - & 0.9510 \\
\hline GST & & & & \\
\hline BRENT & 0.0000 & 0.0337 & 0.5899 & 0.9632 \\
\hline WTI & 0.0000 & 0.0460 & 0.8056 & 0.9470 \\
\hline MME & 0.0000 & 0.0313 & 0.6217 & 0.9649 \\
\hline Normal & & & & \\
\hline BRENT & 0.0093 & 0.0580 & - & 0.9419 \\
\hline WTI & 0.0242 & 0.0619 & - & 0.9348 \\
\hline MME & 0.0070 & 0.0449 & - & 0.9549 \\
\hline LOS & & &
\end{tabular}

Los errores estándar se muestran entre paréntesis.

Fuente: Elaboración propia.

Los valores de la prueba de efectos ARCH sobre los residuales estandarizados se reportan en la Tabla 5, los resultados muestran la ausencia de heterocedasticidad condicional en la serie de los residuales estandarizados para cada uno de los respectivos modelos GARCH.

Tabla 5

Prueba de efectos ARCH-LM para los residuales estandarizados

\begin{tabular}{lrrr}
\hline Series & GARCH-estable & GARCH-GST & \multicolumn{1}{l}{ GARCH-normal } \\
\hline BRENT & 0.7391 & 0.7242 & 0.7637 \\
\hline WTI & 0.0881 & 0.1019 & 0.0696 \\
\hline MME & 0.4056 & 0.4028 & 0.4077 \\
\hline
\end{tabular}

ARCH-LM es la prueba de efectos ARCH (Engle, 1982) considerando 5 rezagos.

Los p-values se muestran entre paréntesis.

Fuente: Elaboración propia. 
De las Tablas 4 y 5, concluimos que los modelos GARCH analizados describen de manera adecuada los clusters de volatilidad condicional, característica empírica de los rendimientos del petróleo, además que los parámetros a1 y b1 satisfacen la condición de estacionariedad e indican un alto grado de persistencia de la volatilidad en los rendimientos.

\section{Estimaciones del VaR}

Como ya se mencionó previamente, en la estimación del VaR, la elección de la distribución apropiada del proceso de innovación es crucial ya que impacta directamente en la calidad de la estimación de los cuantiles requeridos para estimar el riesgo, además, el supuesto sobre la distribución en el modelo GARCH es también clave en el pronóstico del VaR, ya que en base a éste se construyen las funciones de verosimilitud requeridas para estimar los parámetros y se determina la distribución futura del riesgo condicionada a la volatilidad pronosticada.

En el presente documento se estima el VaR a un día considerando tres distribuciones alternativas en el proceso de innovación: estable, t-Student generalizada asimétrica y normal. En la Tabla 6 se observa que las estimaciones del VaR-estable son mayores que las estimaciones del Var-normal y VaR-GST, además es importante señalar que las estimaciones del VaR-normal al 95\% de confianza son mayores que las del VaR-GST, en contraste, para el nivel de confianza del $99 \%$ estas estimaciones muestran un comportamiento opuesto.

Tabla 6

Estimaciones del VaR

\begin{tabular}{lcclrrr}
\hline \multirow{2}{*}{ Series } & \multicolumn{2}{c}{ VaR 95\% } & \multicolumn{3}{c}{ VaR 99\% } \\
\cline { 2 - 7 } & Estable & Normal & GST & Estable & \multicolumn{1}{c}{ Normal } & \multicolumn{1}{c}{ GST } \\
\hline BRENT & -2.2459 & -1.5061 & -1.4040 & -3.8157 & -2.1530 & -2.2040 \\
\hline WTI & -2.0262 & -1.2554 & -1.1841 & -3.0884 & -1.7685 & -1.8482 \\
\hline MME & -1.8504 & -1.1396 & -1.0220 & -3.3294 & -1.6580 & -1.7106 \\
\hline
\end{tabular}

Fuente: Elaboración propia.

\section{Evaluación del desempeño del VaR}

La evaluación del desempeño de predicción del VaR bajo los modelos GARCH-estable, GARCH-GST y GARCH-normal fuera de la muestra se realiza empleando los datos históricos del último año de la muestra para predecir el VaR actual. La Tabla 7 muestra los resultados del estadístico de Kupiec, cuya hipótesis nula H0: $\mathrm{n} / \mathrm{T}=\alpha$, se rechaza con un nivel de significancia $\alpha=1 \%$ si el valor del estadístico excede o es igual al valor crítico de una distribución $\mathrm{Ji}$-cuadrada con un grado de libertad, es decir LRUC $\geq 6.635$. Los valores en negritas indican el modelo con mejor desempeño para estimar el VaR según el estadístico de Kupiec. 
Tabla 7

Estadístico de Kupiec

\begin{tabular}{cccc}
\hline \multicolumn{2}{c}{ Estable } & \multicolumn{1}{c}{ Normal } & GST \\
\hline BRENT & & $19.095(0.000)$ \\
\hline 0.05 & $0.1044(0.747)$ & $13.017(0.000)$ & $1.1644(0.281)$ \\
\hline 0.01 & $5.0051(0.025)$ & $10.734(0.001)$ & \\
\hline WTI & & $22.404(0.000)$ \\
\hline 0.05 & $1.9772(0.160)$ & $22.404(0.000)$ & $1.9772(0.160)$ \\
\hline 0.01 & $1.1644(0.281)$ & $4.3148(0.038)$ & \\
\hline MME & & & $25.876(0.000)$ \\
\hline 0.05 & $1.1644(0.281)$ & $22.404(0.000)$ & $1.1644(0.281)$ \\
\hline 0.01 & $5.0051(0.025)$ & $8.1149(0.004)$ &
\end{tabular}

Los p-values se muestran entre paréntesis.

Fuente: Elaboración propia.

En la Tabla 7 se observan los siguientes resultados:

1. La distribución normal, como se esperaba muestra un deficiente desempeño en la estimación del VaR en ambos niveles de confianza. Lo anterior se debe a que los rendimientos del petróleo estudiados poseen una distribución empírica con colas más pesadas que la normal.

2. De acuerdo con el estadístico de Kupiec, la distribución GST muestra un pobre desempeño en la estimación del VaR al 95\% de confianza, sin embargo muestra resultados excelentes para un nivel de confianza del $99 \%$.

3. La distribución estable presenta un excelente desempeño en la predicción del VaR ya que las tasa de fallas empíricas son estadísticamente iguales a sus valores teóricos.

\section{Conclusiones}

En el sector petrolero, el VaR se ha implementado con el objetivo de cuantificar lo mejor posible los movimientos extremos de los precios del petróleo asociados a un nivel de confianza dado. Esta cuantificación es fundamental no solo en la gestión de riesgos de este sector, sino también en el sector económico y financiero, Sadorsky (1999), afirma que los movimientos extremos en los precios del petróleo repercuten la actividad económica y afectan significativamente los movimientos en el mercado accionario.

Con este propósito, en esta investigación cuantificamos el VaR considerando tres tipos de petróleo (Brent, WTI y MME) y analizamos el desempeño de la estimación del VaR a un día mediante el estadístico de Kupiec considerando modelos GARCH con tres distribuciones 
alternativas en el proceso de innovación: estable, t-Student generalizada asimétrica y normal en un período de alta volatilidad. Los resultados obtenidos señalan que a un nivel de confianza del 99\%, el VaR-estable y VaR-GST presentan un excelente desempeño en la predicción del VaR ya que las tasa de fallas empíricas son estadísticamente iguales a sus valores teóricos.

Sin embargo, los resultados de la evaluación de desempeño del modelo basado en el estadístico de Kupiec señalan que el modelo VaR-estable es un modelo más robusto y preciso para ambos niveles de confianza que los basados en la distribución GST y normal. Este resultado es crucial en el sector financiero, debido a que impacta directamente en la previsión de reservas necesarias para afrontar potenciales pérdidas. En términos globales, esto es de importancia para cualquier agente del sector financiero internacional, ya que estas reservas están en función del nivel de riesgo al que se enfrentan los agentes financieros cuando conforman un portafolio.

Es importante mencionar que sería posible extender esta investigación en un futuro cuantificando el riesgo en el mercado petrolero mediante un modelo multivariado estable, esto con el objetivo de analizar las potenciales interrelaciones entre las volatilidades de los distintos tipos de petróleo.

\section{Referencias}

Barone-Adesi, G., Giannopoulos, K., \& Vosper, L. (1999). VaR without correlations for portfolios of derivative securities. Journal of Futures Markets, 19(5), 583-602. http://doi.org/10.1057/9781137465559.0007

Aloui, C. \& Mabrouk, S. (2010). Value-at-risk estimations of energy commodities via long-memory, asymmetry and fat-tailed GARCH models. Energy Policy, 38(5), 2326-2339. http://doi.org/10.1016/j.enpol.2009.12.020

Bali, T. G. \& Theodossiou, P. (2007). Aconditional-SGT-VaR approach with alternative GARCH models. Annals of Operations Research, 151(1), 241-267.

Bollerslev, T. (1986). Generalized atutoregressive conditional heteroskedasticity. Journal of Econometrics, 31, 307-327.

Cabedo, J. D. \& Moya, I. (2003). Estimating oil price "value at risk" using the historical simulation approach. Energy Economics, 25(3), 239-253. http://doi.org/10.1017/CBO9781107415324.004

Champagnat, N., Deaconu, M., Lejay, A., Navet, N. \& Boukherouaa, S. (2013). An empirical analysis of heavytail behavior of financial data: The case for power laws. Hal. Disponible en: https://hal.inria.fr/hal-00851429

Cheng, W. H. \& Hung, J. C. (2011). Skewness and leptokurtosis in GARCH-typed VaR estimation of petroleum and metal asset returns. Journal of Empirical Finance, 18(1), 160-173. http://doi.org/10.1016/j.jempfin.2010.05.004

Costello, A., Asem, E. \& Gardner, E. (2008). Comparison of historically simulated VaR: Evidence from oil prices. Energy Economics, 30(5), 2154-2166. http://doi.org/10.1016/j.eneco.2008.01.011

De Jesús Gutierrez, R., Ortiz, E., García, O., \& Morales, V. (2016). Medición del riesgo de la cola en el mercado del petróleo mexicano aplicando la teoría de valores extremos condicional. EconoQuantum, 13(2), 77-98.

Dickey, D. \& Fuller, W. (1979). Distribution of the estimators for autoregressive time series with a unit root. Journal of the American Statistical Association, 74, 427-431.

Engle, R. F. (1982). Autoregressive Conditional Heteroskedasticity with Estimates of the Variance of United Kingdom Inflation. Econometria, 50(4), 987-1007. 
Fama, E. F. (1965). The Behavior of Stock-Market Prices. The Journal of Business, 38(1), 34 -105.

Fan, Y., Zhang, Y. J., Tsai, H. T. \& Wei, Y. M. (2008). Estimating "Value at Risk" of crude oil price and its spillover effect using the GED-GARCH approach. Energy Economics, 30(6), 3156 -3171. http://doi.org/10.1016/j. eneco.2008.04.002

Giot, P. \& Laurent, S. (2003). Market risk in commodity markets : a VaR approach. Energy Economics, 25(25), 435-457.

Hamilton, J. D. (1983). Oil and the Macroeconomy since World War II. Journal of Political Economy, 91(2), 228-248. http://doi.org/10.1086/261140

Hang Chan, N., Deng, S. J., Peng, L., \& Xia, Z. (2007). Interval estimation of value-at-risk based on GARCH models with heavy-tailed innovations. Journal of Econometrics, 137(2), 556-576. http://doi.org/10.1016/j. jeconom.2005.08.008

Hansen, B. E. (1994). Autoregressive Conditional Density Estimation. International Economic Review, 35(3), 705730.

Hung, J. C., Lee, M. C. \& Liu, H. C. (2008). Estimation of value-at-risk for energy commodities via fat-tailed GARCH models. Energy Economics, 30(3), 1173-1191. http://doi.org/10.1016/j.eneco.2007.11.004

Jarque, C.M. \& Bera, A.K. (1980). Efficient tests for normality, homoskedasticity and serial independence of regression residuals. Economics Letters, 6, 225-259.

Jorion, P. (2001). Value at Risk, The New Benchmark for Managing Financial Risk. (2nd Edition). McGraw-Hill, United States.

Kupiec, P. H. (1995). Techniques for verifying the accuracy of risk measurement models. Journal of Derivatives, 3(2), 73-84.

Kwiatkowski, D., Phillips, P.C.W., Schmidt, P., Shin, Y. (1992). Testing the null hypothesis of stationarity against the alternative of unit root. Journal of Econometrics, 54, 159-178.

Marimoutou, V., Raggad, B. \& Trabelsi, A. (2009). Extreme Value Theory and Value at Risk: Application to oil market. Energy Economics, 31(4), 519-530. http://doi.org/10.1016/j.eneco.2009.02.005

Morana, C. (2001). A semiparametric approach to short-term oil price forecasting. Energy Economics, 23(3), 325338. http://doi.org/10.1016/S0140-9883(00)00075-X

Nolan, J. P. (1997). Numerical calculation of stable densities and distribution functions. Communications in Statististics. Stochastic Models, 13, 759-774.

Phillips, P.C.B., Perron, P. (1988). Testing for a unit root in time series regression. Biometrika, 75, 335-346

Ruiz-Porras, A. \& Anguiano Pita, J. E. (2016). Modeling the Dynamics, Volatilities and Interrelations of the Mexican , Brent and WTI Oil Returns. Ensayos Revista de Economía, 35(2), 175-193.

Sadeghi, M. \& Shavvalpour, S. (2006). Energy risk management and value at risk modeling. Energy Policy, 34(18), 3367-3373. http://doi.org/10.1016/j.enpol.2005.07.004

Sadorsky, P. (1999). Oil price shocks and stock market activity. Energy Economics, 21(5), 449-469.

Sadorsky, P. (2006). Modeling and forecasting petroleum futures volatility. Energy Economics, 28(4), 467-488. http://doi.org/10.1016/j.eneco.2006.04.005

Shapiro, S.S. and Wilk, M.B. (1965) An Analysis of Variance Test for Normality (Complete Samples). Biometrika, $52,591-611$.

https://doi.org/10.1093/biomet/52.3-4.591

Schwert, G.W. (1989). Why Does Stock Market Volatility Change Over Time?. Journal of Finance, 44, 1115-1153. Taylor, S. (1986). Modeling Financial Time Series. New York, NY: Wiley.

Youssef, M., Belkacem, L., \& Mokni, K. (2015). Value-at-Risk estimation of energy commodities: A long-memory GARCH-EVT approach. Energy Economics, 51, 99-110. http://doi.org/10.1016/j.eneco.2015.06.010 